\title{
It's All About Change: The Antigen-driven Initiation of B-Cell Receptor Signaling
}

\author{
Wanli Liu ${ }^{1}$, Hae Won Sohn ${ }^{1}$, Pavel Tolar², and Susan K. Pierce ${ }^{1}$ \\ ${ }^{1}$ Laboratory of Immunogenetics, National Institute of Allergy and Infectious Diseases, National Institutes of \\ Health, Rockville, Maryland 20852 \\ ${ }^{2}$ National Institute for Medical Research, The Ridgeway, Mill Hill, London, NW7 1AA, United Kingdom \\ Correspondence: spierce@niaid.nih.gov
}

B-cell responses are initiated by the binding of foreign antigens to the clonally distributed $\mathrm{B}$-cell receptors (BCRs) resulting in the triggering of signaling cascades that activate a variety of genes associated with B-cell activation. Although we now understand the molecular nature of the signaling pathways in considerable detail what remains only poorly understood are the mechanisms by which the information that antigen has bound to the BCR ectodomain is transduced across the B-cell membrane to the BCR cytoplasmic domains to trigger signaling. To a large part this gap in knowledge is because of the paucity of techniques to temporally and spatially resolve changes in the behavior of the BCR that occur within several seconds of antigen binding. With the advent of new live-cell imaging technologies we are gaining our first clear views of the events that lead up to the triggering of BCR signaling cascades. These events may provide potential new targets for therapeutic intervention in disease involving hyper or chronic activation of B cells.

Secific, high-affinity antibody responses are the result of processes based on clonal selection (reviewed in Rajewsky 1996). In the absence of antigen, individuals generate a B-cell repertoire in which each $B$ cell expresses a single heavy and light chain gene, the product of somatic recombination of variable and constant region gene segments. Self-reactive B cells are removed from the repertoire and when antigen enters the immune system it selects those B cells expressing BCR's with highest affinity for the antigen. Under the influence of both $T$ cell and innate immune system regulation the antigenselected B cells are induced to differentiate into short-lived antibody producing cells or enter germinal centers where they undergo the molecularly linked processes of somatic hypermutation and isotype switching. Antigen selection within the germinal centers results in highaffinity memory B cells expressing isotype switched BCRs. These memory B cells account, in large part, for the high titered, high affinity IgG antibody responses observed upon re-exposure to antigen. Thus, we presume that $B$ cells are capable of initiating responses to the universe of foreign antigens to which individuals are exposed and do so through mechanisms that are sensitive to the affinity of the BCR for antigen and by which isotype switched BCRs are more effective. Until recently, the events by

Editors: Lawrence E. Samelson and Andrey Shaw

Additional Perspectives on Immunoreceptor Signaling available at www.cshperspectives.org

Copyright (C) 2010 Cold Spring Harbor Laboratory Press; all rights reserved; doi: 10.1101/cshperspect.a002295

Cite this article as Cold Spring Harb Perspect Biol 2010;2:a002295 
W. Liu et al.

which the binding of antigen to the BCRs triggered signaling remained largely unknown due in a large part to the paucity of experimental approaches that were able to provide the spatial and temporal resolution necessary to capture the earliest events that follow the binding of antigens to BCRs that result in triggering the $\mathrm{B}$ cell's signaling cascades. The conventional biochemical techniques that were used so successfully to describe the components of the BCR signaling cascades were too slow to study early events and could not provide spatial information. The application of new live-cell imaging technologies that allow resolution of single molecules over a timeframe of several seconds to the study of antigen-induced B-cell responses is providing the first views of these processes. Here we review progress in understanding the initiation of the BCR signaling using live-cell imaging technologies and how this new knowledge may explain in part the mechanisms that underlie hyper or chronic activation of B cells in autoimmunity and in B-cell cancers.

\section{THE WHO, HOW, AND WHERE OF ANTIGEN PRESENTATION TO B CELLS (BATISTA AND HARWOOD 2009)}

The responses of $\mathrm{B}$ cells to antigens were traditionally studied by providing B cells with multivalent soluble antigens in solution. Batista et al. (Batista et al. 2001) first made the important observation that B cells could be efficiently activated by antigen expressed by antigen presenting cells (APCs). They showed that the interaction of B cells with APCs lead to the formation of a polarized bull's eye like structure in which the BCRs were concentrated in the center, surrounded by the adherence molecule LFA- 1 . This structure was analogous to the immune synapse earlier described for $\mathrm{T}$ cells following interactions with APCs (Fooksman et al. 2010). The description of the B-cell immune synapse by Batista et al. was followed by several studies that used intravital imaging to describe the interaction of B cells with APCs in lymph nodes in vivo. These studies provided evidence that small soluble antigens are able to enter follicles and activate B cells within the follicles
(Pape et al. 2007). Particulate antigens including viruses and immune complexes were observed to be captured by macrophages lining the subcapsular sinuses and transported into the cortex of the lymph node where they were presented to B cells (Carrasco and Batista 2007; Junt et al. 2007; Phan et al. 2007). In addition, $B$ cells were also observed to engage native antigens on lymph node dendritic cells (Qi et al. 2006). These remarkable findings provided a new view of the initiation of antigen-driven BCR signaling in which BCR activation occurred at the interface of the B cell and APCs.

\section{VIEWING B-CELL ACTIVATION ON ANTIGEN-CONTAINING FLUID LIPID BILAYERS}

The observations that B cells were activated by antigen presented by APCs lead Batista and colleagues to investigate $\mathrm{B}$ cells engaging antigen incorporated into fluid planar lipid bilayers as surrogate APCs (Carrasco et al. 2004; Fleire et al. 2006; Weber et al. 2008). They reported $\mathrm{B}$-cell activation on lipid bilayers to be a remarkably dynamic event. They observed that B cells touched the bilayer through membrane protrusions in which the BCRs engaged antigen and formed microclusters. The microclusters that were enriched in tyrosine phosphorylated proteins and were the site of Syk recruitment, actively signaled for the B cells to spread over the bilayer allowing the engagement of additional antigens. Following maximal spreading the B cell actively moved the BCR-antigen microclusters toward the center of the contact area and contracted to form an immune synapse. This dynamic process of spreading and contraction occurred within minutes of the BCR's first contact with the bilayer and was shown to be an important mechanism to allow $\mathrm{B}$ cells to discriminate their affinity for antigen (Fleire et al. 2006). Subsequent studies provided evidence that the requirement for B-cell activation by membrane-associated and soluble antigens were not identical. B-cell activation on antigen-containing bilayers required the B-cell coreceptor, CD19, to be present within the BCR microclusters whereas B-cell activation by 
soluble antigen was CD19-independent (Depoil et al. 2008). These authors suggested that CD19 preferentially partitions into signaling active antigen-BCR-Syk microclusters and amplifies signaling. As described later other fundamental differences between the requirements for B-cell activation by membrane associated versus soluble antigen have been recently described.

\section{THE REQUIREMENT FOR PHYSICAL CROSSLINKING OF BCRS BY MULTIVALENT ANTIGENS}

The BCR is composed of a membrane form of the $\mathrm{Ig}(\mathrm{mIg})$ that alone is not expressed on the cell surface and is unable to initiate signaling. Both surface expression and signaling require the noncovalent association of $\mathrm{mIg}$ with a disulfide-linked heterodimer of $\operatorname{Ig} \alpha$ and $\operatorname{Ig} \beta$ that contain ITAMs in their cytoplasmic domains (Reth 1992) in a $1 \mathrm{mIg}: 1 \operatorname{Ig} \alpha-\operatorname{Ig} \beta$ heterodimer stoichiometry (Schamel and Reth 2000; Tolar et al. 2005). When B cells are provided with antigen the first kinase in the signaling cascades, Lyn, is recruited to and phosphorylates the tyrosines in the $\operatorname{Ig} \alpha / \operatorname{Ig} \beta$ cytoplasmic domains. The question is how is the information that the BCR has bound antigen to its ectodomain translated to a change in the BCR's cytoplasmic domain that can be recognized by Lyn. It is a common observation that monovalent antigens provided in solution are unable to activate $\mathrm{B}$ cells and that activation by antigen in solution requires the binding of BCRs to multivalent antigens, resulting in the clustering, patching, and ultimate capping of BCR-antigen complexes. Such observations lead to the conclusion that B-cell activation requires the physical crosslinking of BCRs by multivalent antigens (Metzger 1992). This crosslinking model for BCR activation fit with the interpretation of $\mathrm{X}$-ray crystallographic studies of Fabs both free and bound to antigen that showed no evidence for a conformational change in the Fab on antigen binding that could be transduced down the mIg to trigger signaling (Poljak 1991). However, these influential structures of Fabs bound to antigens may be misleading in terms of signal transduction. The structure of the BCR has not yet been solved, thus, we do not understand how the mIg ectodomains and the ectodomains of the $\operatorname{Ig} \alpha-\operatorname{Ig} \beta$ complex fit together and how antigen binding might perturb this relationship to transduce signals across the membrane. Thus, until a structure of a complete BCR complex is solved it may be too early to rule out conformational changes in BCRs on antigen binding. Indeed, the more recent crystal structures of intact IgGs provide evidence for extraordinary interdomain flexibility within Igs (Saphire et al. 2002). Additional crystal structures of the Fc of IgE (Wurzburg et al. 2000) and IgG (Radaev et al. 2001; Sondermann et al. 2000) free and bound to Fc receptors revealed a large rearrangement of the Fc upon Fc receptor binding, again indicating the conformational flexibility of the Igs. In collaboration with our National Institutes of Health (NIH) colleague, Peter Sun and members of his research group, we recently carried out structural and functional studies of the $\operatorname{Ig} \alpha-\operatorname{Ig} \beta$ ectodomain and its assembly with the mIg. Our results provided evidence for a structural model for the BCR in which both Ig $\alpha$ and $\operatorname{Ig} \beta$ of the heterodimer bind to the membrane proximal $\mathrm{C} \mu 4$ domain of $\mathrm{mIg}$ through an extensive contact surface involving multiple charged residues (Radaev et al. 2010). It is possible that antigen induced alteration in this interface play a role in transducing information to the cytoplasmic domains.

Nonetheless, in the absence of evidence for a mechanism by which antigen binding to $\mathrm{mIg}$ transduces conformational changes along the chain, the crosslinking model gained traction. Receptor crosslinking by ligands is a common mechanism for receptor activation in other biological receptor systems but for the most part these are monomeric receptors that contain kinases in their cytoplasmic domains, as in the receptor tyrosine kinases, such as the EGF receptor or stably associate with kinases such as Janus kinases in the large family of cytokine receptors (Schlessinger 2000). In these cases, receptor crosslinking brings kinases in the cytoplasmic domains into close proximity allowing cross phosphorylation and receptor activation. In contrast, the BCR has no inherent kinase activity but rather relies on recruitment of Lyn 
W. Liu et al.

following antigen binding. Receptors that rely on ligand-induced crosslinking also differ from the BCR in a second important way, they are activated by one or a small set of structurally related ligands. These receptors have evolved to recognize their ligands and the ligands can reproducibly bring the receptors into a geometry that allows transphosphorylation. In contrast, the BCRs must be able to respond to a universe of foreign antigens of all sizes and shapes with no preconception of what these antigens will be. It is difficult to imagine how antigen binding to BCRs could in all cases result in BCRs crosslinked into a specified geometry. In addition, antibody responses can be generated to small monovalent toxins and to highly mobile cell membrane components such as phospholipids that have no obvious mechanism to crosslink BCRs.

Reth and colleagues (Reth et al. 2000) appreciated this difficulty with the BCR crosslinking model and proposed that in resting cells BCRs self assembled into clusters that antigens disrupt to initiate signaling. Their hypothesis was based in part on biochemical evidence for high molecular weight BCR oligomers in detergent lysates of resting $B$ cells in the absence of antigen (Schamel and Reth 2000). However, recent studies using fluorescent resonance energy transfer (FRET) failed to provide evidence that BCR oligomers exist in the membranes of live resting B cells (Tolar et al. 2005). Nonetheless, the observation of BCR oligomers in detergent solution is of interest, suggesting that BCRs may have an intrinsic propensity to oligomerize.

Our recent studies characterizing the early events in the initiation of B-cell signaling led to the surprising finding that B cells are activated by monovalent antigens but only when the $\mathrm{B}$ cells engaged antigen in fluid lipid bilayers mimicking an APC (Tolar et al. 2009). We observed that the responses of splenic B cells specific for the hapten NIP placed on a fluid lipid bilayer containing $\mathrm{NIP}_{1}$-conjugated peptides attached to fluid lipid bilayers were indistinguishable from B cells responding to lipid bilayers containing the multivalent antigen $\mathrm{NIP}_{14^{-}}$ BSA. In solution the $\mathrm{NIP}_{1}$-peptide, as predicted, had no effect on B cells indicating a qualitative difference between monovalent antigen in solution and monovalent antigen incorporated into fluid lipid bilayers. Neither NIP ${ }_{1}$-peptide in solution nor in a fluid lipid bilayer has the ability to physically crosslink the BCR, and yet monovalent haptenated peptides incorporated into fluid lipid bilayers but not in solution activate $\mathrm{B}$ cells. A fundamental difference between monovalent antigens in solution and in fluid bilayers is the potential of antigen in membranes in to exert a force or torque on the antigen-bound BCR. Such a strain on the BCR could conceivably be transduced into changes that would affect either the mIg ectodomains or the transmembrane domains directly or their relationship with the associated $\operatorname{Ig} \alpha / \operatorname{Ig} \beta$ heterodimer, ultimately resulting in changes in the cytoplasmic domains of the BCR. Of course conformational changes are easier to propose than to prove and direct evidence for a change in the BCR structure upon engagement of antigen on a membrane will likely await the solution of a complete BCR structure.

\section{THE ROLE OF THE BCR'S mlg ECTODOMAIN IN BCR SIGNALING}

Our discovery that B cells responded to monovalent membrane-associated antigens lead us to carry out detailed analyses of the behavior of the BCRs as they first encountered antigen on a fluid lipid bilayer using live-cell imaging at the single molecule level to delineate the mechanism underlying the observed B-cell activation. What we found was consistent with a model we have termed "the conformation-induced oligomerization model" for BCR microclustering (Tolar and Pierce, 2010) (Fig. 1). The key observation from single-molecule imaging was that the BCR when engaging monovalent antigencontaining fluid bilayer stopped. Thus, the binding of monovalent antigen dramatically altered the behavior of the freely diffusing BCR before the formation of microclusters. Two additional results provided evidence that stopping was not the direct result of antigen binding to the BCR but rather that antigen-bound BCRs were mobile in the membrane and only stopped when they encountered other antigen-bound 


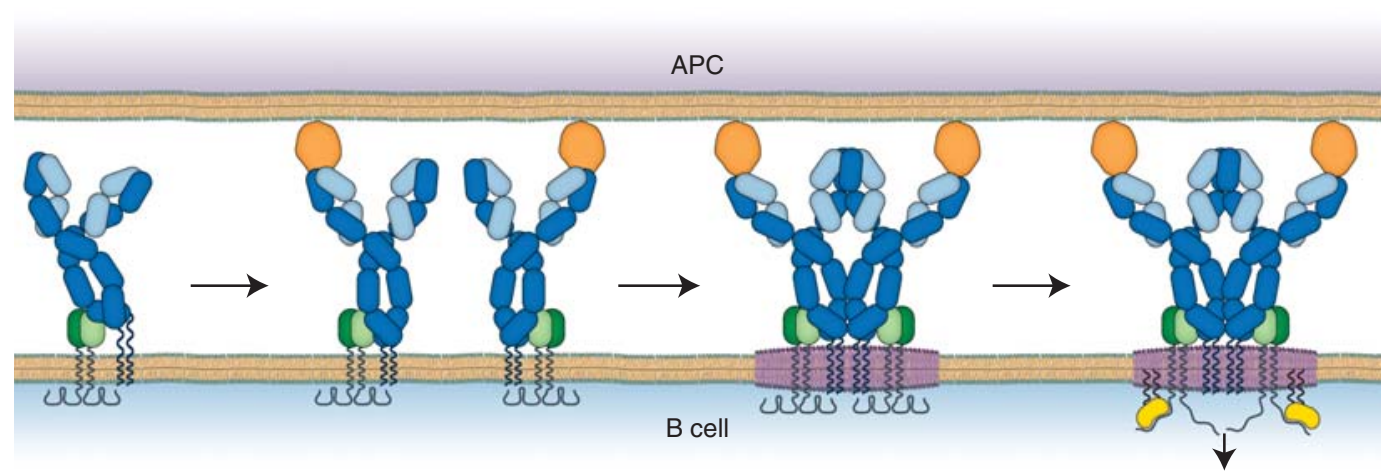

Figure 1. The "conformation-induced oligomerization" model for the initiation of BCR signaling. From left to right: In the absence of antigen the BCR (blue H chain, cyan L chains, green $\operatorname{Ig} \alpha \operatorname{Ig} \beta$ ) is freely diffusing in the B-cell membrane in a conformation that is not permissive for oligomerization. Binding to monovalent antigen on an APC surface reveals an oligomerization face in the $\mathrm{C} \mu 4$ ectodomain of the BCR. The antigen bound BCR is still freely diffusing but stops when it encounters another antigen bound BCR and oligomerizes. The BCR oligomer perturbs the local lipid environment resulting in the coalescence of saturated lipids (in purple) around the oligomer resulting in the simultaneous recruitment of the membrane tethered Lyn (yellow color) and the "opening" of the BCR cytoplasmic domains. Lyn's phosphorylation of the BCR ITAMs triggers the signaling pathways.

BCRs and oligomerized. First, BCR stopping was highly dependent on the concentration of the monovalent antigen in the bilayer. Monovalent antigens diffuse more rapidly in the lipid bilayer than do BCRs in the B-cell membrane so that by imaging the monovalent antigens in bilayers we determined that BCRs bound to and slowed the movement of the antigens (Tolar et al. 2009). However, the antigens stopped only when the concentrations of the antigen were sufficiently high to ensure a high probability that antigen-bound BCRs would encounter each other. Second, imaging the movement of individual $\mathrm{BCR}$ in areas where immobile BCR microclusters had already formed, we observed that BCRs were far more likely to stop when they entered immobile BCR clusters (Tolar et al. 2009). We concluded from these results that monovalent antigen binding altered the BCR in some way that allowed the antigen bound BCR to oligomerize with and form microclusters with other antigen bound BCRs.

Through an extensive mutational analyses we determined that $\mathrm{BCR}$ clustering in response to monovalent membrane antigens is a BCR intrinsic event that does not require a signaling competent $\mathrm{BCR}$ and was mediated by the membrane proximal ectodomains of the $\mathrm{mIg}$, $\mathrm{C} \mu 4$ (Tolar et al. 2009). Thus, BCRs expressing mIgM that contained in their transmembrane domain a $\mathrm{YS} \rightarrow \mathrm{VV}$ mutation allowing the mIgM to be expressed on the plasma membrane alone, in the absence of $\operatorname{Ig} \alpha / \operatorname{Ig} \beta$, stopped and oligomerized in response to monovalent antigen in the lipid bilayer. However, mIgM $(\mathrm{YS} \rightarrow \mathrm{VV})$ that lacked the $\mathrm{C} \mu 4$ ectodomain failed to stop and oligomerize in response to monovalent membrane antigen. We hypothesized that $\mathrm{C} \mu 4$ contained a homo-oligomerization domain that was in some way hidden or blocked and not accessible in the unligated mIgM but became accessible when the mIgM bound antigen on the opposing fluid lipid bilayer (Fig. 1). We reasoned that if this were indeed the case then the $\mathrm{C} \mu 4$ domain expressed alone may spontaneously oligomerize. We found that in contrast to $\mathrm{C} \mu 2-4$ ( $\mathrm{YS} \rightarrow \mathrm{VV}$ ) and $\mathrm{C} \mu 3-4(\mathrm{YS} \rightarrow \mathrm{VV})$ that when expressed on cell surfaces freely diffused in the membrane, $\mathrm{C} \mu 4(\mathrm{YS} \rightarrow \mathrm{VV})$ expressed alone on the cell surface stopped and formed microscopic clusters in the membrane. When expressed with $\operatorname{Ig} \alpha-\operatorname{Ig} \beta$, $\mathrm{C} \mu 4$ was signaling active as measured by the recruitment of Syk and activation of the cells 
W. Liu et al.

to express CD69. Similar results were obtained for $\mathrm{mIgG}$ in which case the membrane proximal C $\gamma 3$ domain was necessary and sufficient for oligomerization. These results changed our thinking considerably about the role of the $\mathrm{mIg}$ in BCR signaling. The mIg was not simply an inert, passive antigen binding domain that provided a means of physical crosslinking of the BCRs but rather a dynamic active component of the BCR.

\section{THE AFFINITY AND ISOTYPE OF THE BCR INFLUENCE EARLY BCR INTRINSIC EVENTS IN THE INITIATION OF SIGNALING}

Immunological memory or the ability to respond more rapidly and robustly to a secondary challenge with antigen is a hallmark of adaptive immunity. For B cells memory responses are characterized by the rapid production of highaffinity, isotype-switched antibodies of which IgGs are the predominant isotypes (Gray 1993). Current evidence indicates that B cells expressing high affinity, isotype switched BCRs are generated in germinal center reactions through the coupled processes of somatic hyper-mutation and switch recombination (McHeyzer-Williams and McHeyzer-Williams 2005). Presumably high affinity, IgG expressing $B$ cells have an advantage and ultimately dominate the memory B-cell compartment. However, the molecular mechanisms by which high-affinity and isotype-switched BCRs confer such an advantage are incompletely understood.

High-affinity B cells have been shown in adoptive transfer experiments in vivo to out compete low affinity B cells for survival, presumably reflecting a competition at the clonal level for limiting quantities of antigen, appropriate niches or $\mathrm{T}$ cell help (Dal Porto et al. 2002; Paus et al. 2006; Phan et al. 2006; Shih et al. 2002a,b; Takahashi et al. 1998). At the level of individual B cells evidence has been provided that signaling through high affinity BCRs is qualitatively different from that through low affinity BCRs (Kouskoff et al. 1998) and more recently that the affinity of $\mathrm{B}$ cells for antigen influence the degree to which B cells spread over antigen containing bilayers allowing increased accumulation of antigen and enhanced responses (Fleire et al. 2006). Concerning the benefit of the IgG- versus IgM-BCRs it appears clear that the cytoplasmic tail of IgG leads to enhanced B-cell responses (Horikawa et al. 2007; Kaisho et al. 1997; Martin and Goodnow 2002; Waisman et al. 2007; Wakabayashi et al. 2002) because of qualitatively different signaling (Horikawa et al. 2007) or through mechanisms that involve the phosphorylation of the mIgG tail promoting the recruitment of Grb2 (Engels et al. 2009).

We asked are the BCR intrinsic events that result in BCR clustering in response to monovalent antigen influenced by the affinity or the isotype of the BCR? To do so cell lines were generated that expressed chimeric BCRs that contained $\mathrm{mIgG}$ or $\mathrm{mIgM}$ engineered to express variable regions that were either high or low affinity for the hapten NIP. We discovered that high affinity BCRs formed immobile, signaling active clusters more efficiently as compared with low affinity BCRs (Fig. 2). Imaging these clusters for $120 \mathrm{~s}$ we observed that as compared to low affinity BCRs clusters, the high affinity clusters grew more rapidly in both fluorescence intensity and in size. Ultimately, the rapid growth in the BCR clusters resulted in enhanced downstream signaling to Syk (Liu et al. 2010).

In addition to the sensitivity of BCR clustering to BCR antigen affinity we observed that independent of affinity IgG-BCRs were more efficient than IgM-BCRs in forming immobile signaling active BCRs (Fig. 3). The IgG-BCRs microclusters also grew in fluorescence intensity and size more rapidly as compared to IgM-BCRs resulting in enhanced downstream signaling. Moreover, the enhanced oligomerization and clustering of the IgG-BCRs in response to membrane associated antigens resulted in significant differences in downstream signaling events measured 10 min later including the recruitment of the Syk (Liu unpublished observation). These observations immediately raised the question: What feature of the IgG-BCR accounted for its ability to oligomerize more rapidly? Earlier studies established an essential role for the cytoplasmic tail of $\mathrm{mIgG}$ in enhancing B-cell responses 


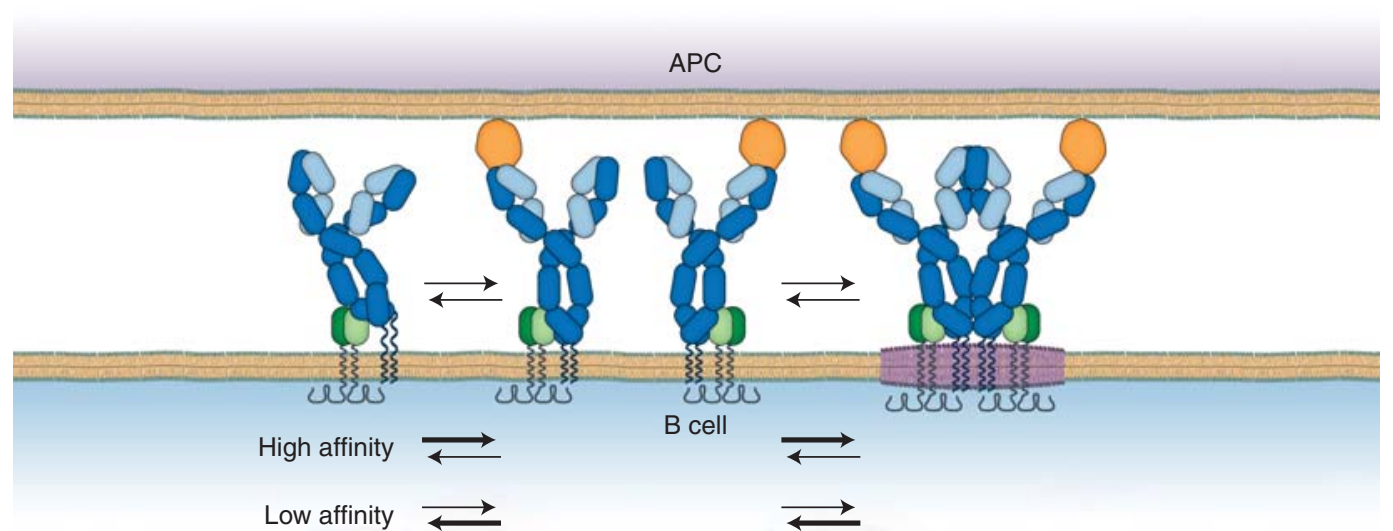

Figure 2. The early intrinsic events in the initiation of signaling are affinity dependent. The BCR transitions reversibly between free, antigen bound and oligomerized states (from left to right). These states are highly sensitive to the affinity of the BCR for antigen. High affinity BCRs are readily driven to oligomers whereas low affinity receptors only inefficiently oligomerize.
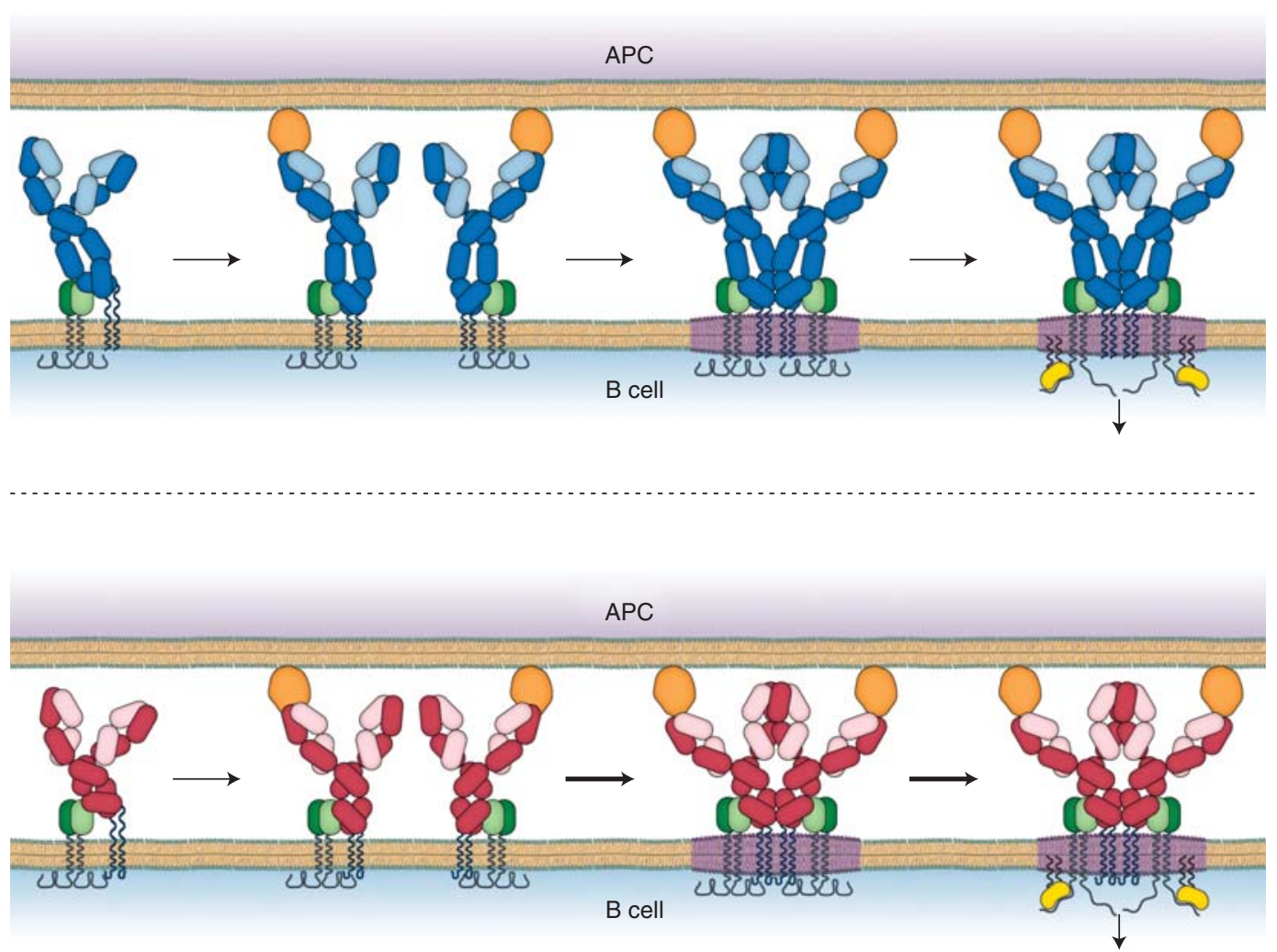

Figure 3. Isotype switched IgG BCRs efficiently initiate early events in BCR signaling. Shown are IgM BCRs (top) and IgG BCRs (bottom). The IgM and IgG BCRs bind antigen similarly in an affinity dependent fashion that brings the BCRs into an oligomerization-permissive conformation. However, because of the properties of its cytoplasmic tail the IgG BCRs oligomerize and initiate signaling more efficiently. 
W. Liu et al.

(Horikawa et al. 2007; Kaisho et al. 1997; Martin and Goodnow 2002; Waisman et al. 2007; Wakabayashi et al. 2002). Our recent studies indicated that the ability of the $\mu$ and $\gamma$ ectodomains to oligomerize was comparable (Tolar et al. 2009) suggesting that the BCR-intrinsic advantage of IgG-BCRs may also be dependent on the mIgG cytoplasmic tail. Indeed, we determined that the enhanced clustering and signaling of IgG BCRs was dependent on the IgG tail. Because these BCR-intrinsic events precede the triggering of signaling cascades, the mIgG tail is presumably functioning in BCR oligomerization by a mechanism distinct from that described by Engels et al. (Engels et al. 2009) involving phosphorylation of the IgG tail.

\section{TRANSLATING BCR OLIGOMERIZATION INTO BCR SIGNALING}

We began this review by asking what changes occur in the BCRs, as the BCRs engage antigen that allows the Lyn to discriminate antigen-free from the antigen-bound receptors? It is possible that the structures of the oligomeric BCR cytoplasmic domains themselves are distinct from that of the monomer. Our earlier published results using quantitative FRET provided evidence that antigen binding resulted in the clustering of the BCR ectodomains and cytoplasmic domains into close molecular proximity and that although the ectodomains remained in close proximity the intracellular domains moved apart, losing FRET (Tolar et al. 2005). We referred to this phenomenon as a transition of the cytoplasmic domains from a "closed" to an "open" form. The temporal relationship between "opening" of the cytoplasmic domains, the phosphorylation of the BCRs by Lyn and the recruitment of Syk could not be resolved leaving the cause-and-effect relationships between these events an open question. Thus, although it is possible that BCR monomers and oligomers are discriminated by Lyn, at present there is little direct experimental support of such a model.

Another possibility for a mechanism by which BCR oligomers and monomers might be discriminated comes from studies of the lipid environment of the BCR monomer and antigen-bound clusters. The unligated BCRs were determined to be more readily solubilized by detergents as compared with antigen-bound BCRs suggesting that the local lipid environment of BCRs changed after antigen binding altering the solubility characteristics (Dykstra et al. 2003). Based on a variety of studies in other receptor systems the differential solubility suggested that the BCRs in resting cells were in relatively disordered membrane microdomains whereas antigen-clustered BCRs were in more ordered membrane microdomains composed of saturated lipids and cholesterol, coined membrane "lipid rafts" (Simons and Toomre 2000). The significance of this observation was that the first kinases in the BCR signal cascade, namely, Src-family kinases were tethered to the membrane by saturated lipids and were concentrated in lipid raft microdomains. Thus, the change in local lipid environment would have the indirect effect of bringing the first kinase into close proximity to the clustered BCR. However, the interpretation of detergent solubility studies are fraught with potential artifacts and if the proposed changes in lipid environment were to be considered seriously as a mechanism for signal transduction, the possibility would need to be tested in intact living cells. To do so we used quantitative FRET analyses of cells that expressed BCRs containing a FRET donor fluorescent protein in their cytoplasmic domains and a series of FRET acceptor fluorescent proteins that were tethered to the cytoplasmic face of the B-cell plasma membrane by saturated or unsaturated lipids. We also studied the association of Lyn itself engineered to contain a FRET acceptor fluorescent protein with the BCR. We observed that on BCR clustering the saturated lipid probe selectively and transiently associated with the BCR microclusters, an interaction that was followed by a prolonged association of the BCR clusters with Lyn (Sohn et al. 2006; Sohn et al. 2008b). The association of the clustered BCRs with Lyn correlated both spatially and temporally with the BCR's cytoplasmic domains coming into a signaling active "open" form. We concluded from these observations that the BCR oligomers 
transiently perturbed the local lipid environment that had the repercussion of bringing the clustered BCRs into close molecular proximity with the Src family kinases promoting proteinprotein interactions between Lyn and the cytoplasmic domains of Ig $\alpha / \operatorname{Ig} \beta$ (Fig. 1) (Tolar et al. 2008). We also suggested that the change in the local lipid environment could have the effect of directly, physically altering the configuration of the receptor's cytoplasmic domains (Fig. 1). Saturated lipids cause thickening and curvature of membranes that could cause the BCR cytoplasmic domains to move to adjust to this change. It is also possible that the cytoplasmic domains of the $\operatorname{Ig} \alpha / \operatorname{Ig} \beta$ heterodimer associate directly with the membrane lipids and that changes in lipids alter this association. Recent studies by $\mathrm{Xu}$ et al. (Xu et al. 2008) showed that the cytoplasmic domain of the T-cell receptor $\mathrm{CD} 3 \varepsilon$ chain associated with the inner leaflet of plasma membrane resulting in the deep insertion of two key tyrosine residues sequestering these from Src kinases in the unligated receptor that were presumably available in the antigen-bound receptor. These results provide an elegant mechanism by which receptor protein-membrane lipid interactions can control the initiation of signaling. The ability of BCR clusters to perturb the local lipid environment resulting in Lyn's association with the BCR may provide a common mechanism by which BCRs physically crosslinked by antigen or engaged by monovalent membrane associated antigens initiate signaling. The BCR clusters that form in response to monovalent antigens on membranes that cannot physically crosslink the BCRs versus multivalent antigen may not be the same in their molecular conformations but may share the common feature of perturbing the membrane, facilitating the interactions of Lyn with the BCR.

\section{REGULATING ANTIGEN-DRIVEN BCR-INTRINSIC EVENTS}

The outcome of signaling through the BCR is regulated by both the developmental stage of the B cell and by a variety of B-cell coreceptors that both promote and attenuate BCR signaling.
This raises the question are the antigen-driven BCR intrinsic events described here regulated either developmentally or by B-cell coreceptors and if so, how? We have begun to address this question by analyzing the effect of a potent B-cell inhibitory receptor, the Fc $\gamma$ RIIB, on early antigen-driven BCR intrinsic events. The FcyRIIB is a well characterized coreceptor that when coligated to the BCR through the binding of immune complexes, recruits the lipid phosphatase SHIP that serves to dampen BCR signaling by reversing the BCR-induced activity of PI3 kinase (Ravetch and Bolland 2001). We determined that the Fc $\gamma$ RIIB had the additional effect of destabilizing BCR oligomers, not by physically disrupting BCR oligomerization but by perturbing the local lipid environment of the BCR oligomer, blocking the BCR's association with saturated lipids and Lyn (Liu et al. 2009; Sohn et al. 2008a) (Fig. 4). Interestingly, an FcyRIIB loss of function mutation in the transmembrane domain of the human protein that is associated with systemic lupus erythematosus, a systemic autoimmune disease (Floto et al. 2005), failed to block the early BCR oligomerization. The transmembrane mutation was previously shown to block the association of FcyRIIB with saturated lipids (Floto et al. 2005). We believe that further studies to elucidate the effect of various coreceptors and the developmental state of the B cell on early BCR intrinsic events may provide new strategies to block hyper-BCR activation in autoimmune diseases.

\section{THE POTENTIAL REPERCUSSIONS OF SPONTANEOUS, CHRONIC BCR OLIGOMERIZATION}

Here we reviewed our current understanding of the molecular mechanisms that underlie the BCR intrinsic events that lead to BCR oligomerization and ultimately to BCR signaling. We showed that mutant BCRs that were composed of mIgs that contained only the membrane proximal domain of $\mathrm{mIgM}, \mathrm{C} \mu 4$, spontaneously oligomerized and signaled (Tolar et al. 2009). This observation raises the question, are there naturally occurring mutations that result 
W. Liu et al.
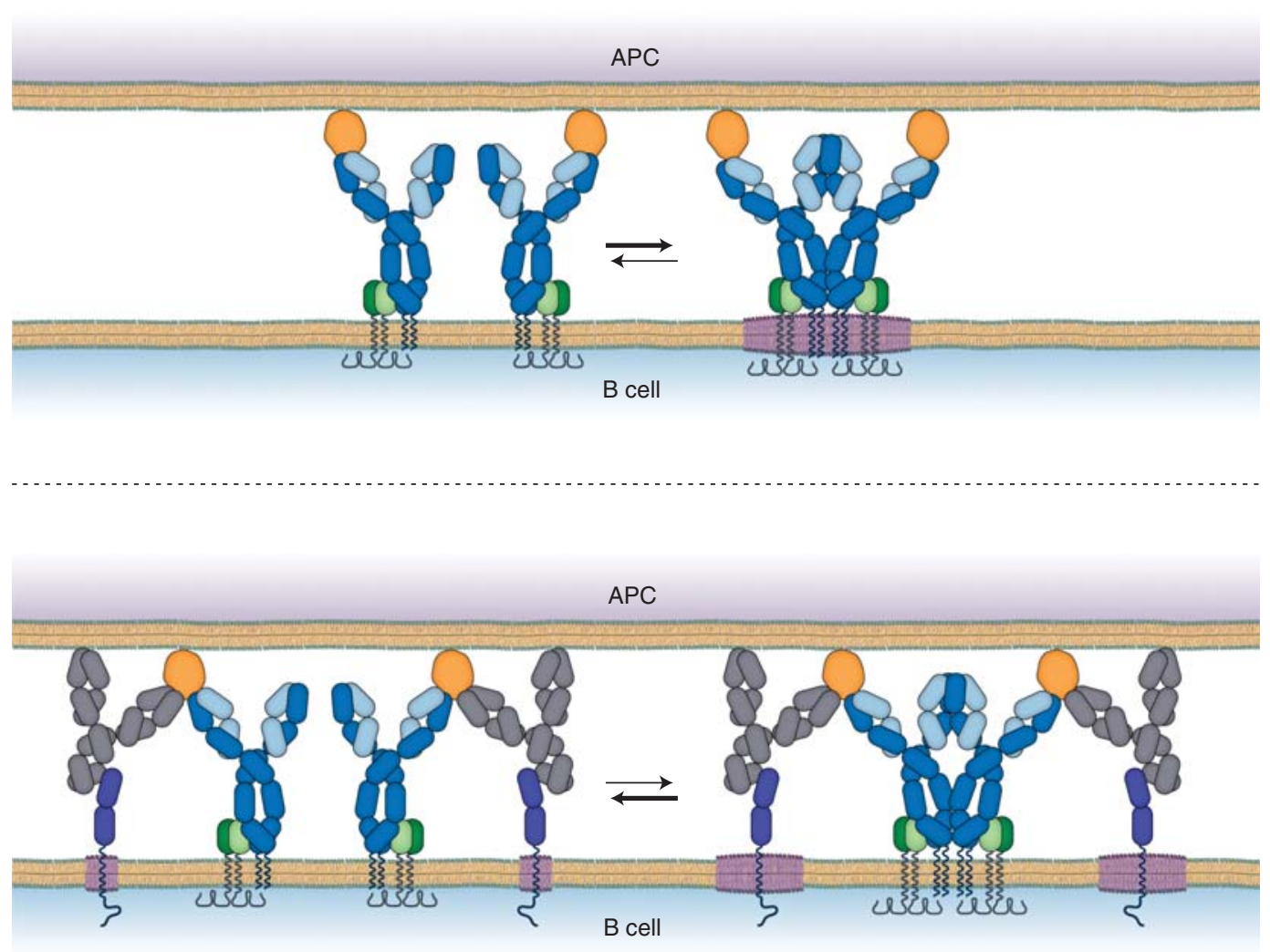

Figure 4. The early BCR intrinsic events in B-cell activation are a novel target for Fc $\gamma$ RIIB inhibition. When coengaged with the FcyRIIB (bottom) through binding of immune complexes (grey colored IgG and orange colored antigen) the Fc $\gamma$ RIIB (bright blue color) blocks the BCR's ability to form stable oligomers (Liu et al. 2009) and associate with raft lipids (Sohn et al. 2008a). Blocking these early events does not require molecular proximity of the cytoplasmic domains of the BCR and Fc $\gamma$ RIIB but relies on the rapid and sustained association of the FcyRIIB with raft lipids.

in spontaneous clustering or activation and if so what are the repercussions of chronic activation? Recently, in collaboration with our NIH colleague, Dr. Louis Staudt and members of his laboratory, we analyzed the behavior of the BCRs on several activated B cell (ABC)-like subtype of diffuse large B-cell lymphomas (DLBCLs) that were dependent on the BCR for their survival. We determined that the BCRs on these tumors formed immobile BCR oligomers within prominent clusters similar to those observed on antigen stimulated normal B cells (Davis et al. 2009). Somatic mutations affecting the cytoplasmic domains of Ig $\alpha$ and $\operatorname{Ig} \beta$ were frequent in these tumors but rarely in other DLBCLs and never in Burkitts or MALT lymphomas. The full sequences of the $\mathrm{mIg}$ of ABC DLBCLs and the correlation with the spontaneous clustering phenotype ought to provide new insights into the mechanisms underlying oligomerization and provide strategies for therapeutic intervention.

\section{SUMMARY}

New live-cell imaging technologies are providing the first views into the extraordinarily dynamic events that accompany the initiation of B cell signaling. Understanding how these events are regulated during development and by coreceptors and how natural mutations affect these events may provide new avenues for therapeutic 
intervention in disease that involve hyper or chronic B-cell activation such as systemic autoimmune diseases and B-cell malignancies.

\section{REFERENCES}

Batista FD, Harwood NE. 2009. The who, how and where of antigen presentation to B cells. Nat Rev Immunol 9: $15-27$.

Batista FD, Iber D, Neuberger MS. 2001. B cells acquire antigen from target cells after synapse formation. Nature 411: 489-494.

Carrasco YR, Batista FD. 2007. B cells acquire particulate antigen in a macrophage-rich area at the boundary between the follicle and the subcapsular sinus of the lymph node. Immunity 27: 160-171.

Carrasco YR, Fleire SJ, Cameron T, Dustin ML, Batista FD. 2004. LFA-1/ICAM-1 interaction lowers the threshold of $\mathrm{B}$ cell activation by facilitating $\mathrm{B}$ cell adhesion and synapse formation. Immunity 20: 589-599.

Dal Porto JMHaberman AMKelsoe G, Shlomchik MJ. 2002. Very low affinity B cells form germinal centers, become memory B cells, and participate in secondary immune responses when higher affinity competition is reduced. J Exp Med 195: 1215-1221.

Davis RE, Ngo VN, Lenz G, Tolar P, Young R, Romesser PB, Kohlhammer H, Lamy L, Zhao H, Yang Y. 2009. Chronic active B cell receptor signaling in diffuse large B cell lymphoma. Nature: in press.

Depoil D, Fleire S, Treanor BL, Weber M, Harwood NE, Marchbank KL, Tybulewicz VL, Batista FD. 2008. CD19 is essential for B cell activation by promoting B cell receptor-antigen microcluster formation in response to membrane-bound ligand. Nat Immunol 9: 63-72.

Dykstra M, Cherukuri A, Sohn HW, Tzeng SJ, Pierce SK. 2003. Location is everything: Lipid rafts and immune cell signaling. Annu Rev Immunol 21: 457-481.

Engels N, Konig LM, Heemann C, Lutz J, Tsubata T, Griep S, Schrader V, Wienands J. 2009. Recruitment of the cytoplasmic adaptor Grb2 to surface IgG and IgE provides antigen receptor-intrinsic costimulation to classswitched B cells. Nat Immunol 10: 1018-1025.

Fleire SJ, Goldman JP, Carrasco YR, Weber M, Bray D, Batista FD. 2006. B cell ligand discrimination through a spreading and contraction response. Science 312: 738741.

Floto RA, Clatworthy MR, Heilbronn KR, Rosner DR, MacAry PA, Rankin A, Lehner PJ, Ouwehand WH, Allen JM, Watkins NA. 2005. Loss of function of a lupusassociated Fc $\gamma$ RIIb polymorphism through exclusion from lipid rafts. Nat Med 11: 1056-1058.

Fooksman DR, Vardhana S, Vasiliver-Shamis G, Liese J, Blair D, Waite J, Sacristan C, Victora G, Zanin-Zhorov A, Dustin ML. 2010. Functional Anatomy of T Cell Activation and Synapse Formation. Annu Rev Immunol 28: 79-105.

Gray D. 1993. Immunological memory. Annu Rev Immunol 11: $49-77$.

Horikawa K, Martin SW, Pogue SL, Silver K, Peng K, Takatsu K, Goodnow CC. 2007. Enhancement and suppression of signaling by the conserved tail of IgG memory-type B cell antigen receptors. J Exp Med 204: 759-769.

Junt T, Moseman EA, Iannacone M, Massberg S, Lang PA, Boes M, Fink K, Henrickson SE, Shayakhmetov DM, Di Paolo NC. 2007. Subcapsular sinus macrophages in lymph nodes clear lymph-borne viruses and present them to antiviral B cells. Nature 450: 110-114.

Kaisho T, Schwenk F, Rajewsky K. 1997. The roles of gamma 1 heavy chain membrane expression and cytoplasmic tail in IgG1 responses. Science 276: 412-415.

Kouskoff V, Famiglietti S, Lacaud G, Lang P, Rider JE, Kay BK, Cambier JC, Nemazee D. 1998. Antigens varying in affinity for the B cell receptor induce differential B lymphocyte responses. J Exp Med 188: 1453-1464.

Liu W, Meckel T, Tolar P, Sohn HW, Pierce SK. 2009. Antigen-induced oligomerization of the $\mathrm{B}$ cell receptor is an early target of FcyRIIB inhibition. J Immunol 184: 1977-1989.

Liu W, Meckel T, Tolar P, Sohn HW, Pierce SK. 2010. Antigen affinity discrimination is an intrinsic function of the $\mathrm{B}$ cell receptor. J Exp Med DOI: 10.1084/jem.20092123.

Martin SW, Goodnow CC. 2002. Burst-enhancing role of the IgG membrane tail as a molecular determinant of memory. Nat Immunol 3: 182-188.

McHeyzer-Williams LJ, McHeyzer-Williams MG. 2005. Antigen-specific memory B cell development. Annu Rev Immunol 23: 487-513.

Metzger H. 1992. Transmembrane signaling: The joy of aggregation. J Immunol 149: 1477-1487.

Pape KA, Catron DM, Itano AA, Jenkins MK. 2007. The humoral immune response is initiated in lymph nodes by $\mathrm{B}$ cells that acquire soluble antigen directly in the follicles. Immunity 26: 491-502.

Paus D, Phan TG, Chan TD, Gardam S, Basten A, Brink R. 2006. Antigen recognition strength regulates the choice between extrafollicular plasma cell and germinal center B cell differentiation. J Exp Med 203: 1081-1091.

Phan TG, Grigorova I, Okada T, Cyster JG. 2007. Subcapsular encounter and complement-dependent transport of immune complexes by lymph node B cells. Nat Immunol 8: $992-1000$.

Phan TG, Paus D, Chan TD, Turner ML, Nutt SL, Basten A, Brink R. 2006. High affinity germinal center B cells are actively selected into the plasma cell compartment. J Exp Med 203: 2419-2424.

Poljak RJ. 1991. Structure of antibodies and their complexes with antigens. Molecular Immunology 28: 1341-1345.

Qi H, Egen JG, Huang AY, Germain RN. 2006. Extrafollicular activation of lymph node B cells by antigen-bearing dendritic cells. Science 312: 1672-1676.

Radaev S, Motyka S, Fridman WH, Sautes-Fridman C, Sun PD. 2001. The structure of a human type III Fcgamma receptor in complex with Fc. J Biol Chem 276: 16469-16477.

Radaev S, Zou Z, Tolar P, Nguyen K, Nguyen AT, Krueger PD, Pierce S, Sun PD. 2010. Structural and functiona studies of Ig alpha/beta and its assembly with the B cell antigen receptor. Structure (in press).

Rajewsky K. 1996. Clonal selection and learning in the antibody system. Nature 381: 751-758. 
W. Liu et al.

Ravetch JV, Bolland S. 2001. IgG Fc receptors. Annu Rev Immunol 19: 275-290.

Reth M. 1992. Antigen receptors on B lymphocytes. Annu Rev Immunol 10: 97-121.

Reth M, Wienands J, Schamel WW. 2000. An unsolved problem of the clonal selection theory and the model of an oligomeric B-cell antigen receptor. Immunol Rev 176: $10-18$.

Saphire EO, Stanfield RL, Crispin MD, Parren PW, Rudd PM, Dwek RA, Burton DR, Wilson IA. 2002. Contrasting IgG structures reveal extreme asymmetry and flexibility. J Mol Biol 319: 9-18.

Schamel WW, Reth M. 2000. Monomeric and oligomeric complexes of the B cell antigen receptor. Immunity 13: $5-14$.

Schlessinger J. 2000. Cell Signaling by Receptor Tyrosine Kinases. Cell 103: 211-225.

Shih TA, Roederer M, Nussenzweig MC. 2002b. Role of antigen receptor affinity in $\mathrm{T}$ cell-independent antibody responses in vivo. Nat Immunol 3: 399-406.

Shih TA, Meffre E, Roederer M, Nussenzweig MC. 2002a. Role of BCR affinity in $\mathrm{T}$ cell dependent antibody responses in vivo. Nat Immunol 3: 570-575.

Simons K, Toomre D. 2000. Lipid rafts and signal transduction. Nat Rev Mol Cell Biol 1: 31-39.

Sohn HW, Pierce SK, Tzeng SJ. 2008a. Live cell imaging reveals that the inhibitory FcgammaRIIB destabilizes B cell receptor membrane-lipid interactions and blocks immune synapse formation. J Immunol 180: 793-799.

Sohn HW, Tolar P, Pierce SK. 2008b. Membrane heterogeneities in the formation of $\mathrm{B}$ cell receptor-Lyn kinase microclusters and the immune synapse. J Cell Biol 182: 367-379.

Sohn HW, Tolar P, Jin T, Pierce SK. 2006. Fluorescence resonance energy transfer in living cells reveals dynamic membrane changes in the initiation of B cell signaling. Proc Natl Acad Sci 103: 8143-8148.

Sondermann P, Huber R, Oosthuizen V, Jacob U. 2000. The 3.2-A crystal structure of the human IgG1 Fc fragment-Fc gammaRIII complex. Nature 406: 267-273.
Takahashi Y, Dutta PR, Cerasoli DM, Kelsoe G. 1998. In situ studies of the primary immune response to (4-hydroxy3-nitrophenyl)acetyl. V. Affinity maturation develops in two stages of clonal selection. J Exp Med 187: 885-895.

Tolar P, Pierce SK. 2010. A conformation-induced oligomerization model for B cell receptor microclustering and signaling. Curr Top Microbiol Immunol 340: $155-169$.

Tolar P, Sohn HW, Pierce SK. 2005. The initiation of antigen-induced B cell antigen receptor signaling viewed in living cells by fluorescence resonance energy transfer. Nat Immunol 6: 1168-1176.

Tolar P, Sohn HW, Pierce SK. 2008. Viewing the antigen-induced initiation of B-cell activation in living cells. Immunol Rev 221: 64-76.

Tolar P, Hanna J, Krueger PD, Pierce SK. 2009. The constant region of the membrane immunoglobulin mediates $\mathrm{B}$ cell-receptor clustering and signaling in response to membrane antigens. Immunity 30: 44-55.

Waisman A, Kraus M, Seagal J, Ghosh S, Melamed D, Song J, Sasaki Y, Classen S, Lutz C, Brombacher F. 2007. IgG1 B cell receptor signaling is inhibited by $\mathrm{CD} 22$ and promotes the development of $\mathrm{B}$ cells whose survival is less dependent on Ig alpha/beta. J Exp Med 204: 747-758.

Wakabayashi C, Adachi T, Wienands J, Tsubata T. 2002. A distinct signaling pathway used by the IgG-containing B cell antigen receptor. Science 298: 2392-2395.

Weber M, Treanor B, Depoil D, Shinohara H, Harwood NE, Hikida M, Kurosaki T, Batista FD. 2008. Phospholipase $\mathrm{C}-\gamma 2$ and Vav cooperate within signaling microclusters to propagate $\mathrm{B}$ cell spreading in response to membranebound antigen. J Exp Med 205: 853-868.

Wurzburg BA, Garman SC, Jardetzky TS. 2000. Structure of the Human IgE-Fc Ce3-Ce4 Reveals Conformational Flexibility in the Antibody Effector Domains. Immunity 13: 375-385.

Xu C, Gagnon E, Call ME, Schnell JR, Schwieters CD, Carman CV, Chou JJ, Wucherpfennig KW. 2008. Regulation of $\mathrm{T}$ cell receptor activation by dynamic membrane binding of the CD3epsilon cytoplasmic tyrosine-based motif. Cell 135: 702-713. 


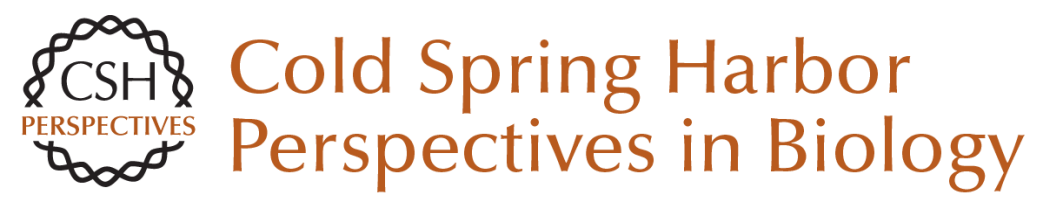

\section{It's All About Change: The Antigen-driven Initiation of B-Cell Receptor Signaling}

Wanli Liu, Hae Won Sohn, Pavel Tolar and Susan K. Pierce

Cold Spring Harb Perspect Biol 2010; doi: 10.1101/cshperspect.a002295 originally published online June 30,2010

\section{Subject Collection Immunoreceptor Signaling}

The Coordination of T-cell Function by

Serine/Threonine Kinases

David Finlay and Doreen Cantrell

ITAM-mediated Signaling by the T-Cell Antigen

Receptor

Paul E. Love and Sandra M. Hayes

Coordination of Receptor Signaling in Multiple Hematopoietic Cell Lineages by the Adaptor

Protein SLP-76

Martha S. Jordan and Gary A. Koretzky

The Cytoskeleton Coordinates the Early Events of B-cell Activation

Naomi E. Harwood and Facundo D. Batista

An Enigmatic Tail of CD28 Signaling

Jonathan S. Boomer and Jonathan M. Green

Mediation of T-Cell Activation by Actin

Meshworks

Peter Beemiller and Matthew F. Krummel
Perspectives for Computer Modeling in the Study

of T Cell Activation Jesse Coward, Ronald N. Germain and Grégoire Altan-Bonnet

Structural Biology of the T-cell Receptor: Insights into Receptor Assembly, Ligand Recognition, and Initiation of Signaling

Kai W. Wucherpfennig, Etienne Gagnon, Melissa J. Call, et al.

Src-family and Syk Kinases in Activating and Inhibitory Pathways in Innate Immune Cells:

Signaling Cross Talk Clifford A. Lowell

The LAT Story: A Tale of Cooperativity,

Coordination, and Choreography Lakshmi Balagopalan, Nathan P. Coussens, Eilon Sherman, et al.

Antigen Receptor Signaling to NF- $\mathrm{KB}$ via

CARMA1, BCL10, and MALT1 Margot Thome, Jean Enno Charton, Christiane Pelzer, et al.

It's All About Change: The Antigen-driven Initiation of B-Cell Receptor Signaling Wanli Liu, Hae Won Sohn, Pavel Tolar, et al.

For additional articles in this collection, see http://cshperspectives.cshlp.org/cgi/collection/

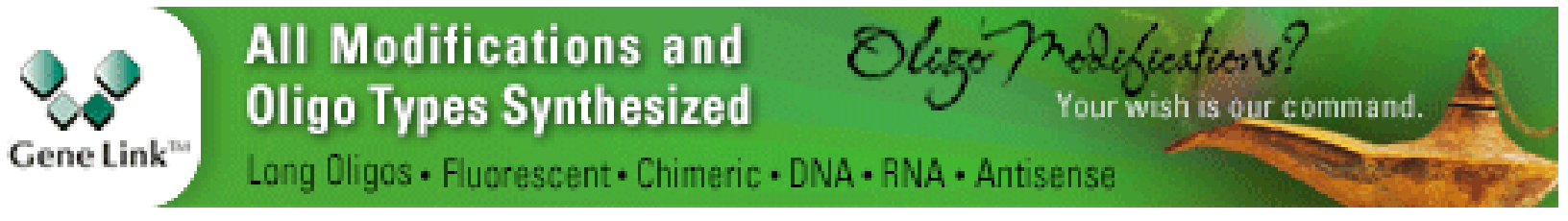


T-Cell Signaling Regulated by the Tec Family

Kinase, Itk

Amy H. Andreotti, Pamela L. Schwartzberg, Raji E. Joseph, et al.

Lipid Signaling in T-Cell Development and

Function

Yina H. Huang and Karsten Sauer
ZAP-70: An Essential Kinase in T-cell Signaling Haopeng Wang, Theresa A. Kadlecek, Byron B. Au-Yeung, et al.

Understanding the Structure and Function of the Immunological Synapse

Michael L. Dustin, Arup K. Chakraborty and Andrey S. Shaw

For additional articles in this collection, see http://cshperspectives.cshlp.org/cgi/collection/

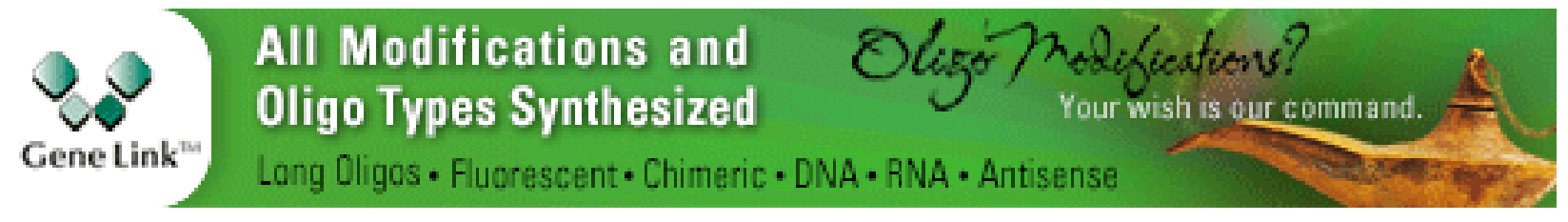

Copyright @ 2010 Cold Spring Harbor Laboratory Press; all rights reserved 